\title{
Force Estimation for Prosthetic Hands Using Surface Electromyography
}

\author{
Xiaobao Deng ${ }^{1,2}$, Xiaogang Duan ${ }^{1,2, *}$ and Hua Deng ${ }^{1,2}$ \\ ${ }^{1}$ State Key Laboratory of High-Performance Complex Manufacturing; \\ ${ }^{2}$ School of Mechanical \& Electrical Engineering, Central South University, Changsha, 410083, China \\ ${ }^{*}$ Corresponding author
}

\begin{abstract}
In order to improve amputees quality of life, electromyogram (EMG) prosthetic hands have been developed to compensate for some daily activities. A force estimation method for prosthetic hands using surface electromyotraphy is proposed in the paper to help amputees. One hand of the subject is tied to imitate amputees. The subjects would grip the force sensor with his healthy hand, while doing the same thing with the tied hand. Meanwhile, EMG signals and force are acquired by LABVIEW. Then a muscle-force model based on stepwise regression method is established .The experiments are carried out to evaluate the grasping force on a single degree of freedom prosthetic hand. The experimental results show that the proposed method is effective.
\end{abstract}

Keywords-prosthetic hand; muscle-force mode; stepwise regression

\section{INTRODUCTION}

As of today, the most common way of feed-forward controlling an active hand prosthesis is via forearm surface electromyotraphy(EMG), a technique by which motor unit activation potentials are read from the amputee's stump skin and then used to command the prosthesis[1]. As sEMG signals are collected from the surface of the skin, the signals pass through numerous tissues before they reaches the surface of the skin and are acquired by the sensors[2]. Hence, they are prone to cross-talk, interference and noise. The sEMG is a temporal and spatially modulated signal[3]. It seems that EMG-based control is not keeping the pace, being limited to a few hand postures or a simple proportional estimate of force.

In the present study, many researchers are focused on the finger grasping pattern more [4], and the grasping force less. In literature [5], using three regression methods (LWPR, ANN, SVM) to estimate grasp force of prosthetic hand. The accuracy of the force reached ninety percent with offline analysis. But calculation is large and online experiment is not easy to be operated. Besides it is only applied to healthy subjects. Excellent results have been shown on healthy subjects, but surprisingly, as far as we know, there is so far just one study on amputees[6].

Inspired by aforementioned researches, we have devised a new experiment. Five healthy people with one hand tied to imitate below-elbow traumatic amputees. They were asked to perform grasp postures with their tied hand at various speeds and forces. Their EMG activity was recorded using two commercially available electrodes, and the desired force was estimated using a force sensor. A muscle-force model was built based on stepwise regression method in order to check how well the required force approximated.

\section{PROBLEM Formulation}

Prosthetic hand control diagram using EMG as shown in Figure 1, where the EMG signal is obtained from the human body. After decoding, grip force and pattern can be obtained. These signals are transmitted to the controller, and to achieve control of the prosthetic hand.

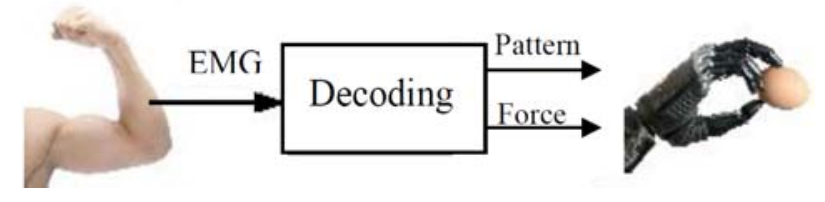

FIGURE I. PROSTHETIC HAND CONTROL DIAGRAM USING EMG

For a prosthetic hand, an important item is how to guarantee grasping stability. Thus, grasp force control is a key element. The amputees can tolerate that there is only a few simple patterns such as a single degree freedom prosthetic hand. But they cannot forgive that prosthetic hand holds an egg but to break it or grasps a hammer but to let it fall.

So far, many researches tended to classify several EMG patterns of single or joint muscle contractions rather than the exerting force of the hand. There methods generally combined feature extraction (AR model[7] and pattern recognition techniques(HMM[8], SMO[9], SVM[10]), can efficiently discriminate particular finger motions or specified hand gestures. Because of merely compressing the resourceful EMG signal into a few muscular contraction modes, the signal into a relatively high level. But this control scheme has insufficient information about how much the force should be applied exactly on the objects.

Some researchers study grasp force. Such as in literature [5], three hand grasp modes were taken into consideration. Prosthetic hands grasped objects by opposing thumb and index finger. Experiments were performed on healthy hands. The algorithm is complex and delay is long. However, the amputees could not grip the force sensor with their phantom limb.

Commercial prosthetic hand is adopting the approach of thresholds control. In other word, when the absolute mean of EMG signal is more than a setting value, the single prosthetic 
hand start moving. The current moment of EMG amplitude is only used. The control method is easy. However, it takes long time to adjust and has insufficient information about EMG signal.

\section{MethodolOGY}

In the paper, the process of griping objects for the amputees is imitated by binding the hand of normal people. A method of muscle model is adopted to estimate muscle force.

According to the principle of muscle model, muscle force is affected by muscle contraction speed and amplitude of EMG signal. When the larger muscle strength is, the smaller the muscle contraction velocity becomes, and the greater the amplitude of EMG signal is. The mathematic model of muscle and force can be obtained:

$$
F(t)=\left[a_{1}(t), a_{2}(t)\right]\left[c_{1}, c_{2}\right]^{T}+\xi
$$

where, $a_{1}(t)$ and $a_{2}(t)$ are status of skeletal muscle. $\xi$ represents the un-modeled effects and random errors in the model. $a_{1}(t)$ and $a_{2}(t)$ are presented :

$$
\begin{aligned}
& \dot{a}_{1}(t)=(0.42 r-1.32 v-1.15) a_{1}(t)+0.75 r \\
& \dot{a}_{2}(t)=(1.3 r-3.07 v-1.15) a_{2}(t)+0.01 r
\end{aligned}
$$

where $r$ is the EMG signal, $v$ is muscle contraction velocity. Thus if the parameters $C_{1}$ and $C_{2}$ were given, the muscle force would be predicted.

As pointed out in [11]," Stepwise regression is a systematic method for adding and removing terms form a multi-linear model based on their statistical significance in a regression.” Apply the stepwise regression method on (1) to determine which mode components make significant contributions to the responses. That is, stepwise regression aims at deriving coefficient vector $\left[c_{1}, c_{2}\right]^{T}$ that indicates the effect size of each explanatory variable in $\left[a_{1}, a_{2}\right]$ on the response variable $F(t)$.

The significance is checked using a double-side t-test on the corresponding parameter. The parameter with the smallest p-value is the most significant term and shall be added accordingly if it is lower than a pre-selected threshold.

If one mode component $a_{\mathrm{j}}$ that was originally in the model does not significantly influence the response $\tilde{F}$, remove this term from the regression model. The significance also can be checked through a double-side t-test. The parameter with the largest p-value is a insignificant term and should be removed accordingly if it is higher than a pre-selected threshold.
The following statistics variable is constructed for performing tests:

$$
t_{i}=\frac{b_{j}}{s e\left(b_{j}\right)}
$$

where $b_{\mathrm{j}}$ is the least squares estimate of with the current regression model. The term $s e\left(b_{\mathrm{j}}\right)$ is the standard error of $c_{\mathrm{j}}$, which can be calculated as the $j^{\text {th }}$ square root value of the diagonal element of the following matrix [12]:

$$
\frac{\sum_{j=0}^{N-1}(\tilde{F}[j]-\hat{F}[j])^{2}}{N-d}\left(A_{d}^{T} A_{d}\right)^{-1}
$$

where $d$ is the degree of freedom, which corresponds to the number of terms already in the regression model. The term $A_{d}$ is the matrix, which includes all the terms $a_{j}$ that are already in the model. The term $\hat{F}[j]$ is the least squares prediction using terms in the regression model.

\section{EXPERIMENTS}

The position of the EMG electrode has a great influence on the EMG signal. Selection of electrode position is noted in literature [13]. Because the thumb plays an important role in the grip process, grasping force should be acquired which can reflect thumb active muscle. Meanwhile, hand is tied in a holding device. Flexor and extensor carpi ulnaris are selected respectively. Commercial surface EMG electrodes (Figure 2.A) are placed on the position as shown in Figure 3. The force sensing resistor (FSR) (Figure 2.B) is pasted on the surface of the silicone glove at thumb finger's tip of prosthetic hand (Figure 3).

Experimental data is acquired by USB-6211 NI at sampling frequency $500 \mathrm{~Hz}$. All of the procedures is implemented in the LABVIEW

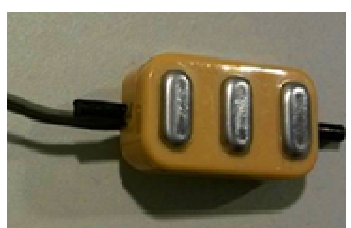

(A) EMG ELECTRODE

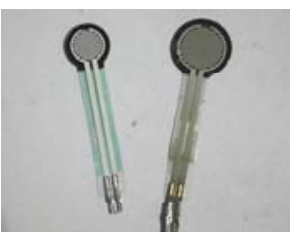

(B) FSR
FIGURE II. EMG AND FORCE SENSORS

Before the start of the experiment, the reliability of the hardware is expected to test, and the subjects were wiped the skin surface by using alcohol to reduce the interference of keratinocytes. Then coat conductive gel on skin. FSR is attached to the left hand of subjects, the right hand imitate the force of the left to grasp an object. Subjects are trained for one minute so that muscle-force model parameters are identified. Then the on-line experiment is carried on. 


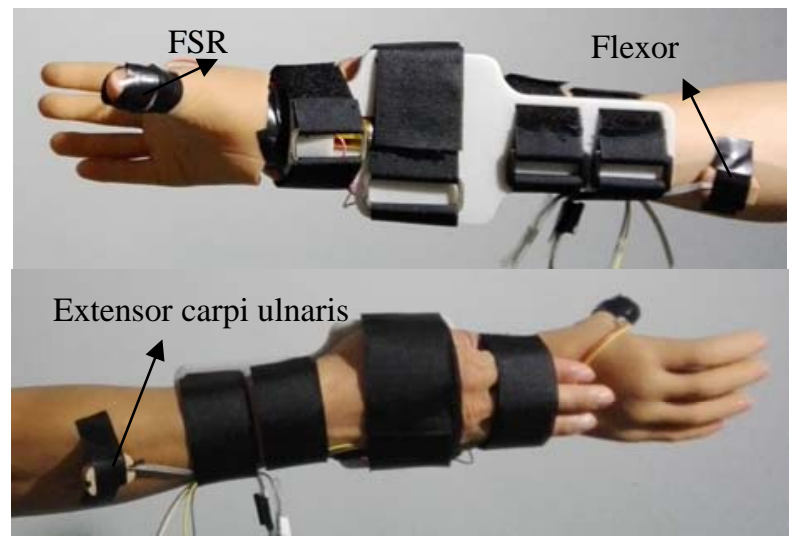

FIGURE III. ELECTRODES AND FSR PLACEMENT

Apply the stepwise regression method to determine the parameters of equation (1). Force estimation performance can be evaluated by two indexes: square error mean (MSE) and correlation coefficients squared (SCC). The result shows that MSE $=0.126941$ and SCC $=87.0355 \%$. We obtain $c 1=15.4273$, c2=-261.2659. Real reference force and prediction are as shown in Figure 4.

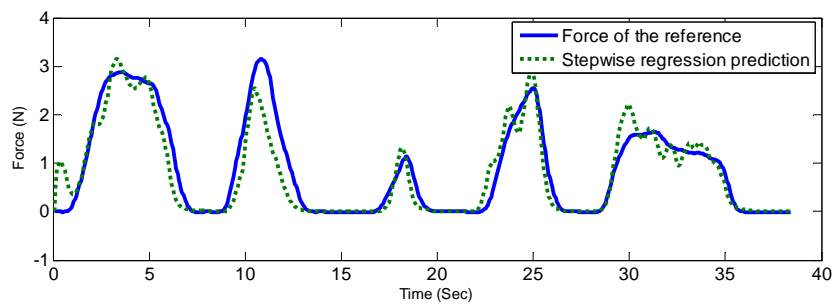

FIGURE IV. COMPARISON BETWEEN THE REFERENCE FORCE AND THE REGRESSION PREDICTION

The parameters are used to estimate muscle force on-line. We grasped a cup of water to drink. The experimental data is as follow. The raw EMG signal is as shown in Figure 5. The force from FSR and prediction from muscle-force mode are shown in Figure 6.

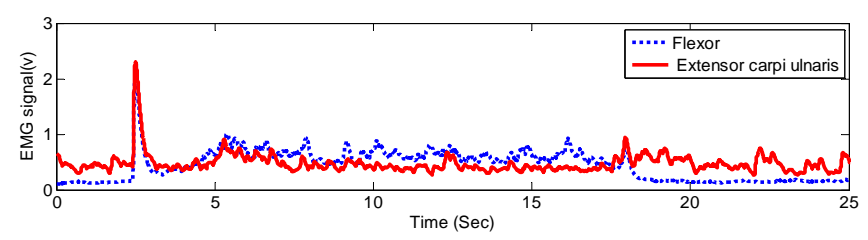

FIGURE V. RAW EMG SIGNAL

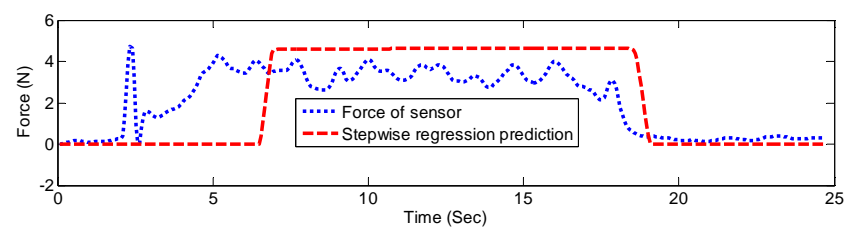

FIGURE VI. FORCE OF SENSOR AND STEPWISE REGRESSION PREDICTION

\section{CONCLUSION}

In the paper, a force estimation method is proposed for prosthetic hands by using surface electromyotraphy. One hand of the subject is tied to imitate amputees. The subjects would grip the force sensor with his healthy hand, while doing the same thing with the tied hand. Then a muscle-force model based on stepwise regression method is established. Experimental results show that the average accuracy of the force decoding by using muscle-force method is more than 80\%. This method can help people with his hand tied successfully grab daily items such as water cup, books. There are some jitter and some errors between the force from EMG signal and the actual force. This problem can be solved by adding some closed loop control method. In the future, double closed loop control method based on force and EMG signal will be researched.

\section{ACKNOWLEDGMENT}

This study was supported by a grant from National Basic Research Program 973 of China (Grant Nos. 2011CB013302)

\section{REFERENCES}

[1] S. Bitzer and P. van der Smagt, "Learning EMG control of a robotic hand: towards active prostheses," in Robotics and Automation, 2006. ICRA 2006. Proceedings 2006 IEEE International Conference on, 2006, pp. 2819-2823.

[2] J. E. Kinesiol, "Introduction to Surface Electromyography," ed, 1998.

[3] E. R. Kandel, J. H. Schwartz, and T. M. Jessell, Principles of neural science vol. 4: McGraw-Hill New York, 2000.

[4] K. Xing, P. Yang, J. Huang, Y. Wang, and Q. Zhu, "A Real-Time EMG Pattern Recognition Method for Virtual Myoelectric Hand Control," Neurocomputing, 2014.

[5] Y. Dapeng, Z. Jingdong, G. Yikun, J. Li, and L. Hong, "Estimation of hand grasp force based on forearm surface EMG," in Mechatronics and Automation, 2009. ICMA 2009. International Conference on, 2009, pp. 1795-1799.

[6] B. E. Boser, I. M. Guyon, and V. N. Vapnik, "A training algorithm for optimal margin classifiers," in Proceedings of the fifth annual workshop on Computational learning theory, 1992, pp. 144-152.

[7] J. Zhao, Z. Xie, L. Jiang, H. Cai, H. Liu, and G. Hirzinger, "EMG control for a five-fingered prosthetic hand based on wavelet transform and autoregressive model," in Mechatronics and Automation, Proceedings of the 2006 IEEE International Conference on, 2006, pp. 1097-1102.

[8] K. R. Wheeler, M. H. Chang, and K. H. Knuth, "Gesture-based control and EMG decomposition," Systems, Man, and Cybernetics, Part C: Applications and Reviews, IEEE Transactions on, vol. 36, pp. 503-514, 2006.

[9] K. Eom, Y. Choi, and H. Sirisena, "EMG pattern classification using SOFMs for hand signal recognition," Soft Computing, vol. 6, pp. 436440, 2002.

[10] P. Shenoy, K. J. Miller, B. Crawford, and R. P. Rao, "Online electromyographic control of a robotic prosthesis," Biomedical Engineering, IEEE Transactions on, vol. 55, pp. 1128-1135, 2008.

[11] MathWorks, MATLAB Statistics Toolbox User's Manual, the MathWorks,2010

[12] N. R. Draper, H. Smith, and E. Pownell, Applied regression analysis vol. 3: Wiley New York, 1966.

[13] J.-U. Chu, I. Moon, Y.-J. Lee, S.-K. Kim, and M.-S. Mun, "A supervised feature-projection-based real-time EMG pattern recognition for multifunction myoelectric hand control," Mechatronics, IEEE/ASME Transactions on, vol. 12, pp. 282-290, 2007. 